\section{Medical/Orthopaedic Management of Rheumatoid Arthritis}

\author{
By R. M. MASON, D.M., F.R.C.P., \\ Physician, Department of Physical Medicine, The London \\ Hospital.
}

Rheumatoid arthritis presents an extremely difficult problem of treatment since it is an inflammatory polyprobritis which pursues a very variable course. Spontaneous remissions are frequent and occur in up to $50 \%$ of hospital remt-patients often leaving no, or only minor, residua. The disease is associated with constitutional disturbance-fever, loss of weight, anaemia, impairment of growth in children and with the presence of nodules. The rheumatoid factor is present in the serum of some 60 to $90 \%$ of adult patients. in patients in which the disease pursues a progressive course the inflammatory changes in the joints produce erosive and destructive lesions - the resulting disability being due to the two factors of inflammatory disease and destructive change. It is very important to assess the separate contributions of these two phenomena before considering appropriate treatment. The inflammatory component has a tendency to diminish in the course of time, whilst the disability due to destructive changes tends to increase.

The three fundamental aspects of treatment comprise physical therapy, drug therapy and orthopaedic measures. No single one of these disciplines should be used in isolation and only a combined team can offer the patient a properly balanced and co-ordinated programme of treatment. PHYSICAL THERAPY

During the inflammatory phase of the disease physical treatment should consist mainly of splinting, prevention of deformity and muscle re-education without producing joint Irauma. Heat has no more than a palliative effect although this may, of course, be useful. In the later stages of the disease, however, physical therapy will be more concerned with the maintenance of joint stability and range of motion. It is only if a proper assessment of the inflammatory and structural changes has been made that physical therapy can be rationalised. Rest as a basic form of treatment should never be overlooked and whilst this may not influence disease activity, functional capacity can often be improved by prolonged, properly controlled, rest. Complete splinting of joints in a plaster cast can be carried out without any risk of loss of range for periods of up to at least a month.

\section{DRUG THERAPY}

Three groups of drugs are available:

(i) Symptomatic.
Aspirin
Butazolidin
Tanderil

(ii) "Anti-Rheumatic."

Gold

Anti-malarials

(iii) Suppressive.

Corticosteroids

Corticotrophin

\section{Symptomatic Drugs:}

Aspirin is probably the safest and most valuable drug of all, but it produces a mild degree of gastric bleeding in almost all subjects, usually amounting to $5 \mathrm{ml}$. blood loss per day. Butazolidin and Tanderil do not produce regular gastrointestinal bleeding but toxicity is more frequent affecting some $25 \%$ of patients. Toxic symptoms develop early rather than late so that prolonged administration is possible in subjects who show no evidence of any idiosyncrasy. Blood dyscrasias may occur but if the dose is restricted to 300 to $400 \mathrm{mg}$. daily these are sufficiently rare as not to form a contra-indication to the use of these drugs.

"Anti-rhuematic", Drugs:

Multi-centre double-blind clinical trials have shown the value of gold therapy in rheumatoid arthritis. The therapeutic effect is manifest after 300 to $400 \mathrm{mg}$. of gold have been administered and once this has been achieved the dose can be reduced or spread out so that prolonged administration can be maintained. Anti-malarials such as the chloroquine group of drugs have also been shown in similar trials to have a distinct anti-rheumatic effect. The only serious toxic effect reported has been retinopathy which may cause a permanent visual defect. But the risk of this complication is slight provided the total duration of administration does not exceed two years.

Suppressive Drugs:

The decision to use steroids in rheumatoid arthritis is a major one and should not be undertaken lightly. These drugs are no more than suppressive and there is no place for short courses of treatment. Unless the physician is prepared to continue administration indefinitely these drugs should not be administered. The dose must be limited to one which can be continued without producing unacceptable side effects, i.e. $10 \mathrm{mg}$. daily of prednisone or its equivalent in a male and $7.5 \mathrm{mg}$. daily in a post-menopausal female. The physician must be prepared to accept a modicum of control in order to achieve prolonged administration. The most important side-effect is the development of arteritis and peripheral neuropathy which reflect a widespread involvement of the arterioles throughout the body and often herald a fatal outcome. The four common serious mistakes in the administration of steroids are as follows:-

(i) Incomplete diagnosis.

(ii) Premature administration.

(iii) Steroid honeymoon.

(iv) Meddlesome dose manipulation.

The four fatal mistakes are as follows:-

(i) Ineffective indoctrination of the patient (including the issue of a steriod card.)

(ii) Excessive continued dosage.

(iii) Abrupt withdrawal of the drug.

(iv) Inadequate "stress" cover.

\section{ORTHOPAEDIC TREATMENT}

Orthopaedic treatment can only be viewed against the background of the medical management of the disease. The major problem is the behaviour of the synovial pannus which invades and destroys the joint acting almost as a locally malignant lesion. In the small joints of the hands early radiological change indicates severe destruction of the joint. Early synovectomy may well be a rewarding procedure in certain circumstances in this situation. Surgical interference of the hand should normally be carried out either very early to prevent destructive changes, or very late as a salvage procedure, but it should not be carried out as a rule during the middle phases of the disease when adaptation is gradually taking place. The more dramatic attrition ruptures of the extensor, or occasionally of the fiexor tendons should not go unrecognised and early repair can be very satisfactory. Surgery to the wrist however can be carried out at any stage. Fusion of the radio-carpal joint together with excision of the lower end of the ulna is an operation almost without penalty and restores to the patient a stable, painless wrist with good pronation and supination. Surgical procedures to the feet such as excision of the metatarsal heads and operations on the toes should be carried out early rather than late. This particularly applies if steroids are to be administered and may, indeed, render them unnecessary. In the knee synovectomy and patellectomy is often a very rewarding procedure, although these operations do not solve the problem of the unstable knee joint. Finally, the problem of the hip joint remains unsolved. Involvement of this joint is the most disabling in rheumatoid arthritis and usually heralds severe loss of functional capacity. Even in the hip joint, however, spontaneous healing may take place.

\section{CONCLUSION}

The Physiotherapist, the Physician and the Orthopaedic Surgeon should all meet at the bedside of the patient with chronic rheumatoid arthritis and work out in collaboration a proper programme of treatment. Only in this way can the patient with progressive rheumatoid arthritis receive adequate treatment. 